\title{
Assessing and Controlling the Size, Morphology and Composition of Supported Bimetallic Catalyst Nanoparticles
}

\author{
Christopher J. Kiely ${ }^{1}$, Qian $\mathrm{He}^{1}$, Ramchandra Tiruvalam ${ }^{1}$, Nikolaos Dimitratos ${ }^{2}$, Michael M.Forde ${ }^{2}$, \\ Meenakshisundaram Sankar ${ }^{2}$ and Graham J. Hutchings ${ }^{2}$ \\ ${ }^{1}$ Department of Materials Science and Engineering, Lehigh University, PA 18015, U.S.A. \\ ${ }^{2}$ Cardiff Catalysis Institute, School of Chemistry, Cardiff University, Wales, U.K.
}

Supported bimetallic nanoparticles are frequently used for applications in catalysis [1]. Ideally we would like to be able to synthesize stable alloy nanoparticles with (i) a well-defined and narrow size range, (ii) very specific morphologies (i.e. random alloy, ordered alloy, core-shell structures) and (iii) closely controlled compositions. In reality, our ability to simultaneously control all of these physical parameters by conventional catalyst preparation methods is still somewhat lacking. We have found that aberration corrected analytical electron microscopy is an excellent way of performing 'quality control' measurements on such bimetallic catalyst systems. In particular, the combination of HAADF imaging and XEDS compositional analysis in the STEM has given us considerable insight into the way in which elemental distributions evolve in these bimetallic catalyst systems during processing and use. Our studies have especially highlighted the need to create simple chemical synthesis protocols which afford a tighter control of composition from particle-to-particle. In this presentation some case studies will be given, using the Au-Pd, Pt-Pd and Au-Pt bimetallic systems as specific examples, to illustrate the complex relationships that can exist between preparation route, compositional homogeneity and catalytic performance.

The most common method of preparing supported Au-Pd bimetallics is via a wet impregnation route in which $\mathrm{HAuCl}_{4}$ and $\mathrm{PdCl}_{2}$ precursor solutions are co-deposited onto a support followed by drying and calcination. This inevitably generates Au-Pd alloy particles having a broad particle size distribution (Fig. 1(a)), with the larger particles being Au-rich and the smaller ones being Pd-rich [2]. Interestingly on oxide supports the Au-Pd particles adopt a Pd-rich shell/Au-rich core structure, whereas on activated carbon they remain as random homogenous Au-Pd alloys. The sheer structural and compositional complexity of the resulting material often hinders the elucidation of meaningful structure-performance relationships in such impregnated bimetallic catalyst systems.

Colloidal synthesis (or sol-immobilization) methods are becoming more popular because they can deliver much better control over particle size [3]. However, the alloy particles so generated suffer the added complication of being coated with organic ligands and still exhibit very significant compositional inhomogeneities. For example, random alloy $\mathrm{Au}-\mathrm{Pd}$ and Pt-Pd particles prepared by sol-immobilization exhibit a systematic size-dependent composition in which the larger particles are Pd-rich and smaller ones are Pd-deficient (i.e. the exact opposite trend to that displayed by analogous materials prepared by wet impregnation). Sol-immobilization methods also have a potential advantage in that they can be deliberately manipulated to allow the preparation of 'designer' core-shell bimetallic morphologies. However, in practice we now know that the particle size distribution of the initial 'seed' colloid that forms the core, can lead to drastic variations in shell thickness (and hence overall composition) from particle-to-particle. Furthermore secondary nucleation events and thermal diffusion effects can also cause further severe deviations away from the expected nominal alloy composition. 
To overcome these materials processing limitations relating to size, morphology and composition control, we have developed two new synthesis protocols for generating supported bimetallic alloys. The first, which we call modified impregnation [4], is a subtle but important variation on the conventional wet impregnation technique, in which excess anion ligands are added during the precursor introduction stage in order to better disperse and mix both metal components. This is followed by a simple heat treatment in a reducing atmosphere, to remove any core-shell variants and solely generate the more desirable random alloy morphologies. The second method, known as chemical vapour impregnation (CVI) [5], involves the direct mixing of suitable organometallic precursor compounds with the support material, followed by a thermal decomposition step within a sealed tube. The supported alloy materials prepared by both the modified impregnation and CVI routes (Figs. 1(b) and(c) respectively) have a smaller average particle size and significantly improved compositional uniformity from particle-toparticle, which ultimately results in a higher activity and stability, as compared to catalysts prepared via the conventional impregnation or sol-immobilization methods.

\section{References:}

[1] M. Sankar, N. Dimitratos, P.J. Miedziak, P.P. Wells, C.J. Kiely and G.J. Hutchings, Chem. Soc. Rev., 41, (2012), 8099-8139.

[2] G.J. Hutchings and C.J. Kiely, Accounts Chemical Research, 46, (2013), 1759-1772.

[3] R.C. Tiruvalam, J. Pritchard, N. Dimitratos, J.A. Lopez-Sanchez, J.K. Edwards, A.F. Carley

G.J. Hutchings and C.J. Kiely, Faraday Discussions, 152, (2011), 63-86.

[4] M. Sankar, M. Morad, J. Pritchard J, Q. He, J.K. Edwards, S.H. Taylor, A.F. Carley, D.W. Knight, C.J. Kiely and G.J. Hutchings, ACS Nano, 6, (2012), 6600-6613.

[5] M.M. Forde, L. Kesavan, M. Izham bin Saiman, Q. He, N. Dimitratos, J.A. Lopez-Sanchez, R.L. Jenkins, C.J. Kiely and G.J. Hutchings, ACS Nano, 8, (2014), 957-969.

[6] The authors wish to thank Drs A.A. Herzing, J.A. Lopez-Sanchez, J.K. Edwards, A.F. Carley, M. Morad, J. Pritchard and L. Kasevan for their valuable contributions to this work.
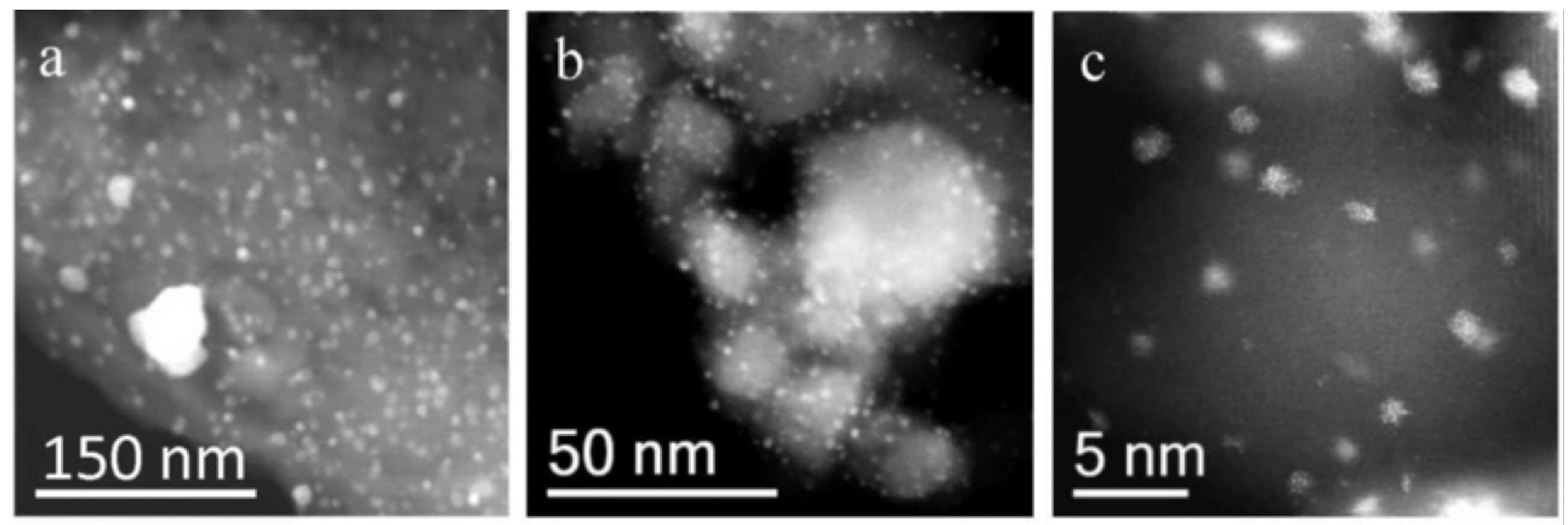

Figure 1. HAADF STEM images of bimetallic Au-Pd nanoparticles supported on $\mathrm{TiO}_{2}$ prepared by (i) conventional wet impregnation, (ii) modified impregnation and (iii) chemical vapour impregnation. 